\title{
POINCARÉ DUALITY AND PERIODICITY, II. JAMES PERIODICITY
}

\author{
JOHN R. KLEIN AND WILLIAM RICHTER \\ To Bruce Williams on his sixtieth birthday
}

\begin{abstract}
Let $K$ be a connected finite complex. This paper studies the problem of whether one can attach a cell to some iterated suspension $\Sigma^{j} K$ so that the resulting space satisfies Poincaré duality. When this is possible, we say that $\Sigma^{j} K$ is a spine.

We introduce the notion of quadratic self duality and show that if $K$ is quadratically self dual, then $\Sigma^{j} K$ is a spine whenever $j$ is a suitable power of two. The powers of two come from the James periodicity theorem.

We briefly explain how our main result, considered up to bordism, gives a new interpretation of the four-fold periodicity of the surgery obstruction groups. We therefore obtain a relationship between James periodicity and the four-fold periodicity in $L$-theory.
\end{abstract}

\section{INTRODUCTION}

This paper is extends the results of [KR]. Recall that the spine of a Poincaré duality space $X$ of dimension $d \geq 3$ is a finite CW complex $K$ of dimension $\leq d-1$ such that $X$ is homotopy equivalent to $K \cup_{\beta} D^{d}$, in which $\beta: S^{d-1} \rightarrow L$ is the attaching map for the top cell of $X$ ([W1, 2.4], [W2]). The pair $(K, \beta)$ is unique up to homotopy type.

We shall consider here the problem of deciding when a finite complex is stably the spine of some Poincare space:

Question. Let $K$ be a connected based finite complex. When is some $j$-fold suspension $\Sigma^{j} K$ the spine of some Poincare space $X$ ?

A question like this appears in the problems section of the proceedings of the 1982 Northwestern homotopy theory conference $[\mathrm{H}]$. It is not hard to construct spaces $K$ answering the question in the negative. Nevertheless, we will see that the question does have a solution for

Date: March 4, 2022.

1991 Mathematics Subject Classification. Primary: 57P10, 57Q45 Secondary: 55Q25, 55P91.

The author is partially supported by the NSF. 
certain interesting class of spaces. Roughly, these spaces will be "very self Spanier-Whitehead dual" in a sense that we will explain.

Our first main theorem says that if $K$ is already a spine of an $X$ and the latter has trivial Spivak fibration, then $\Sigma^{j} K$ for infinitely many $j$ are also spines. More precisely, let us call a Poincaré space $X=K \cup_{\beta} D^{d}$ stably parallelizable if its Spivak normal fibration is trivializable. This is equivalent to the statement that the attaching map $\beta: S^{d-1} \rightarrow K$ is stably null homotopic, and also to the statement that the top cell of $X$ stably falls off.

Let us recall that the Adams number $\phi(\ell)$ is the number of of positive integers $n \leq \ell$ such $n$ is congruent to $0,1,2,4$ modulo 8 .

Theorem A. Assume $\ell \geq 2$. Suppose $K$ is the spine of a Poincaré space $X^{d}$ whose top cell falls off after $\ell$ suspensions.

Then there is a Poincaré space $Y^{d+2 j}$ with spine $\Sigma^{j} K$, where $j=$ $2^{\phi(\ell-1)}$. Furthermore, the top cell of $Y$ splits off after $\ell$ suspensions.

Remarks. (1). The proof will show that $Y$ is functorial in $\beta$ and the choice of null homotopy of $\Sigma^{\ell} \beta$.

(2). Although they have different dimensions, $X$ and $Y$ have the same Euler characteristics and signatures. If we ignore gradings, then the intersection pairings of $X$ and $Y$ are isomorphic.

(3). In a previous paper [KR] we considered the case $\ell=1$ and showed that the analogous statements are true provided one takes $j=2$.

(4). One can apply the theorem iteratively to produce a sequence of Poincaré spaces $X_{s}$ with spine $\Sigma^{s j} X$.

If $K$ fails to be a spine, then one can still give a partial solution to the basic question in terms of Spanier-Whitehead duality. Let

$$
D_{2} K:=\left(E \mathbb{Z}_{2}\right)_{+} \wedge_{\mathbb{Z}_{2}} K^{\wedge 2}
$$

be the quadratic construction, i.e., the reduced homotopy orbits of $\mathbb{Z}_{2}$ acting by transposition on the smash product $K \wedge K$. Note that $D_{2}$ is a functor on the category whose objects are based spaces and whose morphisms are stable maps. In what follows we use the notation $A \rightarrow B$ to denote a stable map of spaces from $A$ to $B$.

A stable map

$$
S^{d} \rightarrow D_{2} K
$$

is said to be a quadratic duality if the composition

$$
S^{d} \rightarrow D_{2} K \stackrel{\operatorname{tr}}{\mapsto} K \wedge K
$$


is an $S$-duality map, where the second displayed map is the transfer associated with the action of $\mathbb{Z}_{2}$ on $K \wedge K$. (Recall that an $S$-duality $u: S^{n} \rightarrow A \wedge B$ is a stable map such that the operation $f \mapsto\left(f \wedge \mathrm{id}_{B}\right) \circ u$ gives an isomorphism $\{A, E\} \cong\left\{S^{n}, E \wedge B\right\}$, where $\{A, E\}$ is the abelian group of homotopy classes of stable maps from $A$ to $E$.) Our notion of quadratic duality is a space level version of a construction given by Ranicki in the category of chain complexes $[R]$.

Theorem B. The following statements are equivalent.

(1) There exists a quadratic duality map $S^{d} \rightarrow D_{2} K$.

(2) There exists a stably parallelizable Poincaré duality space $X^{d+2 j}$ with spine $\Sigma^{j} K$ for some non-negative integer $j$.

Theorem $\mathrm{B}$ can be refined to give explicit information about the integers $j$ for which the statement (2) holds. We say that that $K$ is $j$-periodic for some positive integer $j$ if there is an integer $c$ and a sequence of Poincare complexes $X_{1}, X_{2}, \ldots$ such that the spine of $X_{i}$ is $\Sigma^{c+i j} K$. If no such pair exists, call $K$ aperiodic.

Examples. (1). The sphere $S^{k}$ is aperiodic: since there are only finitely many homotopy classes having Hopf invariant one, there are only finitely many complexes of the form $S^{p} \cup_{\beta} D^{n}$ satisfying Poincaré duality.

(2). The wedge $S^{k} \vee S^{k}$ is 1-periodic, since $S^{j} \vee S^{j}$ is the spine of $S^{j} \times S^{j}$ for every $j$.

(3). The spine of the Stiefel manifold $V_{2}\left(\mathbb{R}^{2 j+3}\right)$ of two-frames in $\mathbb{R}^{2 j+3}$ is $\Sigma^{2 j} \mathbb{R} P^{2}$. Consequently, $\mathbb{R} P^{2}$ is 2 -periodic. It is known not to be 1 periodic, even though $\Sigma \mathbb{R} P^{2}$ is the spine of a five manifold (cf. [KR]).

\section{Let}

$$
D_{2}^{\ell} K=S_{+}^{\ell-1} \wedge_{\mathbb{Z}_{2}} K^{\wedge 2}
$$

where $\mathbb{Z}_{2}$ acts antipodally on $S^{\ell-1}$ (observe that $D_{2}^{\infty} K=D_{2} K$ contains $\left.D_{2}^{\ell} K\right)$.

An $\ell$-refined quadratic duality for $K$ is stable map

$$
S^{d} \rightarrow D_{2}^{\ell} K
$$

such that the composite

$$
S^{d} \rightarrow D_{2}^{\ell} K \stackrel{\subsetneq}{\rightarrow} D_{2} K
$$

is a quadratic duality. Since the the inclusion $D_{2}^{\ell}(K) \rightarrow D_{2}(K)$ is $\ell$-connected, it follows that any quadratic duality factors through an $\ell$-refined one when $\ell$ is sufficiently large. In fact, if $K$ is $r$-connected, then $\ell$ can be taken to be $d-2 r-1$. 
Theorem C. Assume $\ell \geq 2$. Then the following statements are equivalent

(1) There is an $\ell$-refined quadratic duality $S^{d} \rightarrow D_{2}^{\ell} K$.

(2) There is a Poincaré space $Y^{d+2 j}$ with spine $\Sigma^{j} K$ and whose top cell falls off after $\ell$-suspensions, where $j$ is some non-negative integer multiple of $2^{\phi(\ell-1)}$.

If we combine Theorems $\mathrm{A}$ and $\mathrm{C}$ we obtain

Corollary D. Assume $\ell \geq 2$. Then the complexes $K$ satisfying Theorem $\square$ are $2^{\phi(\ell-1)}$-periodic.

If $\ell=1$, then such $K$ are 2-periodic.

Corollary E. Assume $K$ is $r$-connected, $d=2 r+2$ and there is a quadratic duality $S^{d} \rightarrow D_{2}(K)$. Then $K$ is 2-periodic.

The relationship with surgery theory. Let $f: X^{d} \rightarrow S^{d}$ be a Poincaré normal map, that is, the pullback of the stable normal bundle of $S^{d}$ is identified with the Spivak normal bundle of $X$; this is the same thing as equipping $X$ with a stable fiber homotopy trivialization $b$ of its Spivak normal fibration. Poincaré normal maps of this kind arise from stably parallelizable Poincaré spaces $X=K \cup D^{n}$ by collapsing $K \subset X$ to a point. Up to homotopy, every normal map $f: X \rightarrow S^{d}$ is represented in this way.

One is typically interested in the obstruction to deciding when $(f, b)$ is normally cobordant to a homotopy equivalence. If $d$ is odd, then there is no such obstruction (this uses [K1]).

If $d=2 s$ is even, the surgery obstruction $\sigma(f, b)$ is defined and lives in the $L$-group $L_{d}=\mathbb{Z} /\left(1+(-1)^{s-1}\right)$. Poincare embedding theorems for spheres [K2] can be used to show that the surgery obstruction is the complete obstruction when $d \geq 6$. A version of Wall realization also shows that any element of $L_{d}$ comes from a Poincaré normal map. Consequently, the bordism group of normal maps $X^{d} \rightarrow S^{d}$ is in bijection with $L_{d}$ when $d=2 s \geq 6$ (this statement is implicit in [HV]; I intend to publish a homotopy theoretic proof of it in the near future).

Furthermore, one can do surgery to represent any element of the normal bordism group by a normal map $f: X^{d} \rightarrow S^{d}$ which is $s$-connected. The spine $K$ of $X$ is then $(s-1)$-connected, and by Poincaré duality, it has the homotopy type of a CW complex of dimension $\leq s$. Consequently, $K$ has the homotopy type of a finite wedge of $s$-spheres, and so, using Corollary E, one sees that $K$ is 2-periodic. We therefore obtain a sequence of stably parallelizable Poincaré spaces $\left\{X^{d+4 j}\right\}_{j \geq 0}$ such that the spine of $X^{d+4 j}$ is $\Sigma^{2 j} K$. 
Consider the associated Poincaré normal maps $\left(f_{j}, b_{j}\right): X^{d+4 j} \rightarrow$ $S^{d+2 j}$. These have surgery obstructions $\sigma\left(f_{j}, b_{j}\right) \in L_{d+4 j}=L_{d}$. It can be seen from the constructions appearing in our proof that all of these surgery obstructions coincide. It follows that periodicity in the sense of this paper corresponds to the four-fold periodicity in surgery theory. However, our interpretation of the periodicity is different from the usual one: the periodicity operator for $L$-theory is usually described by taking cartesian product with $\mathbb{C} P^{2}$.

Outline. Section 2 gives a criterion for recognizing Poincaré duality on those spaces whose top cell falls off. Section 3 discusses James periodicity. Section 4 describes a $\mathbb{Z}_{2}$-equivariant version of the Whitehead product map. Section 5 contains the proof of Theorem A. Section 6 proves Theorems $\mathrm{B}$ and $\mathrm{C}$. Section 7 is an appendix proving a composition formula for the stable Hopf invariant.

We will be working in the category of based spaces having the homotopy type of a based CW complex. A stable map from $X$ to $Y$ is a map $\Sigma^{\infty} X \rightarrow \Sigma^{\infty} Y$ of associated suspension spectra. We will use the notation $X \rightarrow Y$ to denote such maps.

We use the notion of Poincaré complex defined by Wall [W1]: $X$ is said to be a Poincaré duality space of dimension $d$ if there is a pair $([X], \mathcal{L})$ consisting of a rank one local coefficient system on $X$ and a class $[X] \in H_{d}(X ; \mathcal{L})$ such that the cap product homomorphism $\cap[X]: H^{*}(X ; M) \rightarrow H_{d-*}(X ; \mathcal{L} \otimes M)$ is an isomorphism, where $M$ ranges over all systems of local coefficients.

Acknowledgements. We are indebted to Andrew Ranicki for $L$-theory related discussions, to Ralph Cohen clarifying James periodicity and to Fred Cohen for discussions surrounding the equivariant Whitehead product.

\section{A CRIterion For Poincaré DUALity}

Let $X$ be a space of the form $K \cup_{\beta} D^{d}$, for some attaching map $\beta: S^{d-1} \rightarrow K$. We will assume that $K$ is a connected, based CW complex of dimension $<d$.

Assume $\beta$ is stably null homotopic. A choice of null homotopy $\eta$ then amounts to map of pairs

$$
\left(D^{d}, S^{d-1}\right) \rightarrow(Q K, K)
$$

where $Q K=\Omega^{\infty} \Sigma^{\infty} K$. Let

$$
h: Q K \rightarrow Q D_{2} K
$$


be the stable Hopf invariant [C, p. 62] . As the composite $K \rightarrow Q K \rightarrow$ $Q D_{2} K$ has a preferred null homotopy, $h$ actually determines a map of pairs

$$
(Q K, K) \rightarrow\left(Q D_{2} K, C K\right)
$$

where $C K$ is the reduced cone on $K$. We will also denote this map of pairs by $h$. Taking the composite, we have a map

$$
\left(D^{d}, S^{d-1}\right) \rightarrow(Q K, K) \rightarrow\left(Q D_{2} K, C K\right)
$$

which determines a homotopy class of map of quotients

$$
S^{d} \rightarrow Q D_{2} K
$$

or equivalently a homotopy class of stable map

$$
\delta(\beta, \eta): S^{d} \rightarrow D_{2} K \text {. }
$$

Proposition 2.1. The space $X$ is Poincaré duality space of dimension $d$ if and only if the composite

$$
\operatorname{tr}_{K} \circ \delta(\beta, \eta): S^{d} \rightarrow K \wedge K
$$

is an S-duality map.

Proof. The pair $\beta$ and $\eta$ determine a stable map $\rho: S^{d} \rightarrow X_{+}$and stable retraction $\phi: X_{+} \rightarrow K$. Then $X$ will satisfy Poincaré duality if and only if the composite

$$
S^{d} \stackrel{\rho}{\rightarrow} X_{+} \stackrel{\Delta}{\rightarrow} X_{+} \wedge X_{+}
$$

is an $S$-duality. It is known that the latter coincides with composite

$$
\operatorname{tr}_{X_{+}} \circ h(\rho): S^{d} \rightarrow D_{2}\left(X_{+}\right) \rightarrow X_{+} \wedge X_{+}
$$

(cf. [R], [C, p. 62]). Observe that $K$ is the effect of removing the bottom and top cells of $X_{+}$, and these cells are dual to each other. Consequently, Poincaré duality for $X$ is equivalent to $S$-duality for $(\phi \wedge \phi) \circ \operatorname{tr}_{X_{+}} \circ h(\rho)$.

Observe also that $\phi$ is compatible with transfers, in the sense that

$$
(\phi \wedge \phi) \circ \operatorname{tr}_{X_{+}} \simeq \operatorname{tr}_{K} \circ D_{2}(\phi) .
$$

Hence, Poincaré duality holds for $X$ if and only if $\operatorname{tr}_{K} \circ D_{2}(\phi) \circ h(\rho)$ is an $S$-duality.

If we apply the composition formula for $h$ (cf. Appendix) applied to the null homotopic stable map $\phi \circ \rho$, we see that

$$
D_{2}(\phi) \circ h(\rho)=-h(\phi) \circ \rho .
$$

Consequently, $\operatorname{tr}_{K} \circ D_{2}(\phi) \circ h(\rho)$ coincides with $\operatorname{tr}_{K} \circ h(\phi) \circ \rho$ up to sign. We will be done if we can show that the stable composite

$$
h(\phi) \circ \rho: S^{d} \rightarrow X_{+} \rightarrow D_{2}(K)
$$


coincides with $\delta(\beta, \eta)$. The point is that $h(\phi)$ restricted to $K_{+}$is null homotopic in a preferred way, so we have a factorization of $h(\phi) \circ \rho$ as

$$
S^{d} \rightarrow X_{+} \rightarrow X_{+} / K_{+}=D^{d} / S^{d-1} \rightarrow D_{2} K \text {. }
$$

The composite of the first two of these maps is clearly the identity . Finally, the last map $D^{d} / S^{d-1} \rightarrow D_{2} K$ coincides with $\delta(\beta, \eta)$.

\section{JAMES PERIOdicity}

The quotient

$$
\mathbb{R} P_{m}^{n}:=\mathbb{R} P^{n} / \mathbb{R} P^{m-1},
$$

is standard notation for truncated real projective space. The periodicity theorem of James states

$$
\mathbb{R} P_{m+j}^{n+j} \simeq \Sigma^{j} \mathbb{R} P_{m}^{n}
$$

whenever $j$ is a suitable power of two [J], [Mh]. We will be primarily interested in the case $m=0$. The proof in this instance follows from the computation of the order of the canonical line bundle in the reduced real $K$-theory of $\mathbb{R} P^{n}$. We will reformulate the precise statement below.

Let $\alpha$ denote the involution of $\mathbb{R}^{1}$ given by $t \mapsto-t$; this is sign representation of $\mathbb{Z}_{2}$, and is sometimes denoted by $\mathbb{R}^{\alpha}$. Let $S(\ell \alpha)$ be the unit sphere of $\mathbb{R}^{\ell \alpha}$; this is just $S^{\ell-1}$ with antipodal action. Let $S^{\ell \alpha}$ be the one point compactification of $\mathbb{R}^{\ell \alpha}$.

The total space of the canonical line bundle $\xi_{\ell}$ over $\mathbb{R} P^{\ell-1}$ is

$$
S(\ell \alpha) \times_{\mathbb{Z}_{2}} \mathbb{R}^{\alpha},
$$

where bundle projection to $\mathbb{R} P^{\ell-1}$ is given by first factor projection. By Adams [A], the order of $\xi_{\ell}$ is $j:=2^{\phi(\ell-1)}$, in the sense that $j$ is the smallest positive integer for which $j \xi_{\ell}$ is stably trivial.

If we write $\ell-1=8 d+r$, where $0 \leq r<8$, then

$$
j=\phi(8 d+r) \geq \phi(8 d)=2^{4 d}>8 d+r=\ell-1 .
$$

It follows that $j \xi_{\ell}$ is trivial, since it is a stably trivial rank $j$-bundle over a CW complex of dimension $<j$. In particular, there is an isomorphism of fiberwise one point compactified sphere bundles

$$
S(\ell \alpha) \times_{\mathbb{Z}_{2}} S^{j \alpha} \cong \mathbb{P}^{\ell-1} \times S^{j}
$$

over $\mathbb{P}^{\ell-1}$. This isomorphism preserves zero sections.

Suppose $A$ is a based $\mathbb{Z}_{2}$-space. Then $S(\ell \alpha) \times_{\mathbb{Z}_{2}} A \rightarrow \mathbb{P}^{\ell-1}$ is a fibration with section. The isomorphism of sphere bundles above induces, by taking fiberwise smash products, an equivalence of fibrations with section

$$
S(\ell \alpha) \times_{\mathbb{Z}_{2}}\left(S^{j \alpha} \wedge A\right) \simeq S(\ell \alpha) \times_{\mathbb{Z}_{2}}\left(\Sigma^{j} A\right)
$$


Collapsing zero sections, we obtain

Lemma 3.1. Assume $\ell \geq 2$. For $j=2^{\phi(\ell-1)}$ and a based $\mathbb{Z}_{2}$-space $A$, there is a weak equivalence of based spaces

$$
S(\ell \alpha)_{+} \wedge_{\mathbb{Z}_{2}}\left(S^{j \alpha} \wedge A\right) \simeq S(\ell \alpha)_{+} \wedge_{\mathbb{Z}_{2}}\left(\Sigma^{j} A\right) .
$$

Remark 3.2. If we take $A=S^{0}$, the statement gives a homotopy equivalence $\mathbb{R} P_{j-1}^{\ell+j-1} \simeq \Sigma^{j} \mathbb{R} P^{\ell-1}$.

Corollary 3.3. With $\ell$ and $j$ as above and $K$ a based space, there is a weak equivalence

$$
\Sigma^{j-1}\left(S(\ell \alpha)_{+} \wedge_{\mathbb{Z}_{2}}\left(S^{j \alpha} \wedge K^{\wedge 2}\right) \simeq \Sigma^{2 j-1} D_{2}^{\ell}(K) .\right.
$$

After suspending once, this becomes an equivalence

$$
D_{2}^{\ell}\left(\Sigma^{j} K\right) \simeq \Sigma^{2 j} D_{2}^{\ell}(K)
$$

which is compatible with transfers, in the sense that the diagram

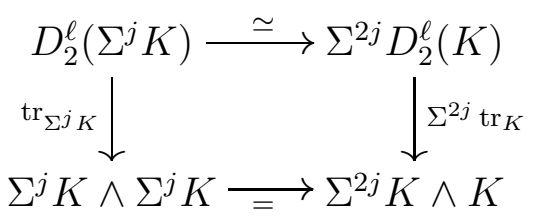

homotopy commutes.

Proof. For the first part, use take $A=\Sigma^{j-1} K \wedge K$ with evident action and apply [3.1. As for the second part, the equivalence $D_{2}^{\ell}\left(\Sigma^{j} K\right) \simeq$ $\Sigma^{2 j} D_{2}^{\ell}(K)$ arises from an isomorphism of bundles over $\mathbb{P}^{\ell-1}$ which can be pulled back along $S(\ell \alpha)$ to obtain an isomorphism of double covers of the total spaces. Compatibility with transfers now follows.

Remark 3.4. When $\ell=1$ we can take $j=2$ and the conclusions of 3.3 will hold.

\section{A generalized Whitehead Product}

The Whitehead product $w: \Sigma K \wedge K \rightarrow \Sigma K$ is obtained from the cofiber sequence

$$
\Sigma K \wedge K \stackrel{W}{\longrightarrow} \Sigma K \vee \Sigma K \stackrel{\subset}{\longrightarrow} \Sigma K \times \Sigma K
$$

by taking the composition of $W$ with the fold map.

We will construct below an equivariant (weak) map

$$
\tilde{\omega}: S^{\alpha} \wedge(K \wedge K) \rightarrow \Sigma K
$$

where the target has trivial involution. Unequivariantly, this map will coincide with $w$ up to homotopy. 
Construction of $\tilde{\omega}$. Recall that the join $K * K$ is the quotient space of $K \times K \times[0,1]$ in which $(x, y, 0)$ and $\left(x^{\prime}, y, 0\right)$ are identified, and also $(x, y, 1)$ and $\left(x, y^{\prime}, 1\right)$ are identified for all $x, x^{\prime}, y, y^{\prime} \in K$. The map $(x, y, t) \mapsto(t,(x, y))$ defines a homotopy equivalence

$$
K * K \stackrel{\sim}{\rightarrow} \Sigma K \wedge K .
$$

If we give $K * K$ the involution $(x, y, t) \mapsto(y, x, 1-t)$ and $S^{\alpha} \wedge K \wedge K$ the involution $(t,(x, y)) \mapsto(1-t,(y, x))$, then the displayed map is is equivariant, so we have an equivariant weak equivalence. We henceforth identify

$$
K * K \simeq S^{\alpha} \wedge K \wedge K
$$

Consider the map

$$
K * K \rightarrow \Sigma(K \vee K)
$$

defined by the formula

$$
(x, y, t) \mapsto \begin{cases}(4 t, x, *), & t \in[0,1 / 4], \\ (4 t-1, *, y), & t \in[1 / 4,1 / 2], \\ (3-4 t, x, *), & t \in[1 / 2,3 / 4], \\ (4-4 t, *, y), & t \in[3 / 4,1] .\end{cases}
$$

If we give $\Sigma(K \vee K)$ the involution which is trivial on the suspension coordinate, but transposes summands (i.e., $(t,(x, y)) \mapsto(t,(y, x)))$ then the map is $\mathbb{Z}_{2}$-equivariant. If we compose with the fold map, we get a $\mathbb{Z}_{2}$-equivariant map

$$
K * K \rightarrow \Sigma K
$$

which we will take the liberty of writing as an equivariant map

$$
\tilde{\omega}: S^{\alpha} \wedge(K \wedge K) \rightarrow \Sigma K,
$$

even though the map $K * K \rightarrow \Sigma K$ does not obviously factor through the equivariant weak equivalence $K * K \rightarrow S^{\alpha} \wedge(K \wedge K)$.

The map $\omega_{\ell}$. For each $\ell \geq 1$, we will define a map

$$
\omega_{\ell}: S(\ell \alpha)_{+} \wedge_{\mathbb{Z}_{2}} S^{\alpha} \wedge(K \wedge K) \rightarrow \Sigma K .
$$

Let $S(\ell \alpha)_{+} \rightarrow S^{0}$ be the map which collapses $S(\ell \alpha)$ to a point. Smash this map with $\tilde{\omega}$. This results in a $\mathbb{Z}_{2}$-equivariant map

$$
S(\ell \alpha)_{+} \wedge\left(S^{\alpha} \wedge(K \wedge K) \rightarrow \Sigma K\right.
$$

Finally, $\omega_{\ell}$ is the factorization of this last map over orbits; here we are using the fact that target has trivial involution.

Let

$$
E_{\ell}: \Sigma K \rightarrow \Omega^{\ell} \Sigma^{\ell}(\Sigma K)
$$


be the adjoint to the identity.

Lemma 4.1. The composite $E_{\ell} \circ \omega_{\ell}$ has a preferred null homotopy. Furthermore, if $C$ denotes the cone on $S(\ell \alpha)_{+} \wedge_{\mathbb{Z}_{2}}\left(S^{\alpha} \wedge K^{\wedge 2}\right)$, then the associated commutative square

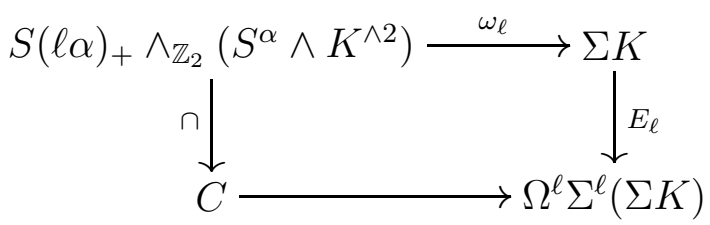

is $(4 r+7)$-cocartesian when $K$ is $r$-connected.

Proof. The second filtration $F_{2}(\Sigma K)$ of the configuration space model for $\Omega^{\ell} \Sigma^{\ell}(\Sigma K)$ sits in a pushout square

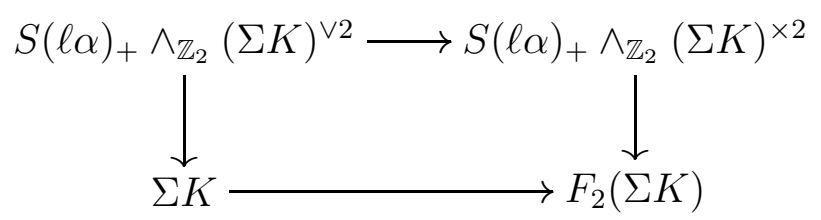

in which the horizontal arrows are inclusions and the left vertical arrow is the evident fold map ([My], $[\mathrm{S}]$ ). The top horizontal arrow sits in a cofiber sequence

$$
S(\ell \alpha)_{+} \wedge_{\mathbb{Z}_{2}}\left(S^{\alpha} \wedge K^{\wedge 2}\right) \rightarrow S(\ell \alpha)_{+} \wedge_{\mathbb{Z}_{2}}(\Sigma K)^{\vee 2} \rightarrow S(\ell \alpha)_{+} \wedge_{\mathbb{Z}_{2}}(\Sigma K)^{\times 2}
$$

where the first map is given by smashing $\tilde{\omega}$ with the identity of $S(\ell \alpha)_{+}$ and then taking orbits. It follows that there is also a cofiber sequence

$$
S(\ell \alpha)_{+} \wedge_{\mathbb{Z}_{2}}\left(S^{\alpha} \wedge K^{\wedge 2}\right) \rightarrow \Sigma K \rightarrow F_{2}(\Sigma K) .
$$

Moreover, the composite

$$
S(\ell \alpha)_{+} \wedge_{\mathbb{Z}_{2}}\left(S^{\alpha} \wedge K^{\wedge 2}\right) \rightarrow \Sigma K \rightarrow F_{2}(\Sigma K) \rightarrow \Omega^{\ell} \Sigma^{\ell}(\Sigma K)
$$

coincides with $E_{\ell} \circ \omega_{\ell}$, and is therefore null-homotopic. The other part of the lemma also follows from the observation that the map $F_{2}(\Sigma K) \rightarrow$ $\Omega^{\ell} \Sigma^{\ell}(\Sigma K)$ is $(4 r+7)$-connected.

Corollary 4.2. Let $j=2^{\phi(\ell-1)}$, and use $\Sigma^{j} K=\Sigma^{j-1}(\Sigma K)$ in place of $\Sigma K$. Then using 3.3, the map $\omega_{\ell}$ may be rewritten in the form

$$
\Sigma^{2 j-1} D_{2}^{\ell} K \rightarrow \Sigma^{j} K
$$




\section{Proof of Theorem $\mathrm{A}$}

Let $X=K \cup_{\beta} D^{d}$, and suppose $\Sigma^{\ell} \beta: S^{d+\ell-1} \rightarrow \Sigma^{\ell} K$ comes equipped with a null homotopy $\eta$. Then we have a map of pairs

$$
\phi(\eta, \beta):\left(D^{d}, S^{d-1}\right) \rightarrow\left(\Omega^{\ell} \Sigma^{\ell} K, K\right)
$$

Let $E_{\ell}: K \rightarrow \Omega^{\ell} \Sigma^{\ell} K$ be adjoint to the identity, and let

$$
h_{\ell}: \Omega^{\ell} \Sigma^{\ell} K \rightarrow \Omega^{\ell} \Sigma^{\ell} D_{2}^{\ell} K
$$

be the Hopf invariant. Then $h_{\ell} \circ E_{\ell}$ is preferred null homotopic, so $h_{\ell}$ may be considered as a map of pairs

$$
\left(\Omega^{\ell} \Sigma^{\ell} K, K\right) \rightarrow\left(\Omega^{\ell} \Sigma^{\ell} D_{2}^{\ell} K, C K\right)
$$

Then by taking quotients $h_{\ell} \circ \phi(\eta, \beta)$ induces a map

$$
f: S^{d} \rightarrow \Omega^{\ell} \Sigma^{\ell} D_{2}^{\ell} K \text {. }
$$

By the material in $\S 2$, the composite

$$
S^{d} \stackrel{f}{\longrightarrow} \Omega^{\ell} \Sigma^{\ell} D_{2}^{\ell} K \subset Q D_{2} K \rightarrow K \wedge K
$$

is an $S$-duality.

Taking the adjoint of $f$, we obtain a map

$$
S^{d+\ell} \rightarrow \Sigma^{\ell} D_{2}^{\ell} K
$$

Suspend this map $(2 j-\ell-1)$-times to obtain a map

$$
S^{d+2 j-1} \rightarrow \Sigma^{2 j-1} D_{2}^{\ell} K
$$

Since $j=2^{\phi(\ell-1)}$ we can apply Lemma 4.2 to compose this last map with $\omega_{\ell}: \Sigma^{2 j-1} D_{2}^{\ell} K \rightarrow \Sigma^{j} K$ to get a map

$$
\gamma: S^{d+2 j-1} \rightarrow \Sigma^{j} K \text {. }
$$

Theorem $\mathrm{A}$ is a direct consequence of the following.

Proposition 5.1. Let $Y=\left(\Sigma^{j} K\right) \cup_{\gamma} D^{d+2 j}$ be the mapping cone of $\gamma$. Then $Y$ is a Poincaré duality space of dimension $d+2 j$ whose top cell splits off after $\ell$ suspensions.

Proof. Recall that the attaching map $\gamma$ is defined so as to factor through $\omega_{\ell}$. Furthermore, by 4.1 and 4.2 we have a commutative diagram

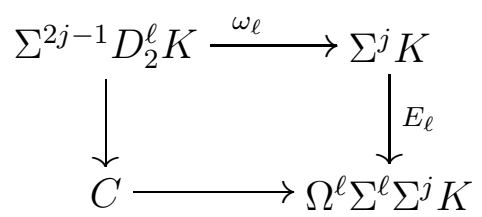

with $C$ contractible. Hence the top cell of $Y$ splits off after $\ell$ suspensions. It remains to show that $Y$ satisfies Poincaré duality. 
The diagram together with $\gamma$ yields maps of pairs

$$
\left(D^{d+2 j}, S^{d+2 j-1}\right) \rightarrow\left(\Omega^{\ell} \Sigma^{\ell} \Sigma^{j} K, \Sigma^{j} K\right) \stackrel{h_{\ell}}{\rightarrow}\left(\Omega^{\ell} \Sigma^{\ell} D_{2}^{\ell}\left(\Sigma^{j} K\right), C \Sigma^{j} K\right) .
$$

The induced map of quotients determines a homotopy class of map

$$
S^{d+2 j} \rightarrow \Omega^{\ell} \Sigma^{\ell} D_{2}^{\ell}\left(\Sigma^{j} K\right) .
$$

Taking the adjoint and using the identification $D_{2}^{\ell}\left(\Sigma^{j} K\right) \simeq \Sigma^{2 j} D_{2}^{\ell}(K)$ of 3.3, we obtain a map

$$
S^{d+2 j+\ell} \rightarrow \Sigma^{2 j+\ell} D_{2}^{\ell} K
$$

which is just the $2 j$-fold suspension of the map $f: S^{d+\ell} \rightarrow \Sigma^{\ell} D_{2} K$. Since the transfer applied to $f$ is an $S$-duality map, it now follows by 2.1 that $Y=\left(\Sigma^{j} K\right) \cup_{\gamma} D^{d+2 j}$ is a Poincaré duality space.

\section{Proof of Theorems B And C}

Theorem $\mathrm{B}$ is a direct consequence of Theorem $\mathrm{C}$.

Proof that (2) implies (1) in Theorem [C. Suppose that $X=\left(\Sigma^{j} K\right) \cup_{\beta}$ $D^{n}$ is a Poincaré space such that $\Sigma^{j} \beta$ is null homotopic, where $j=$ $2^{\phi(\ell-1)}$. Then we have a commutative diagram

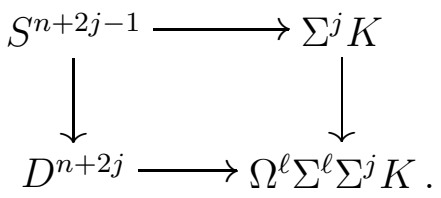

We can then take the composition of the maps of pairs

$$
\left(D^{n+2 j}, S^{n+2 j-1}\right) \rightarrow\left(\Omega^{\ell} \Sigma^{\ell} \Sigma^{j} K, \Sigma^{j} K\right) \stackrel{h_{\ell}}{\rightarrow}\left(\Omega^{\ell} \Sigma^{\ell} D_{2}\left(\Sigma^{j} K\right), C \Sigma^{j} K\right)
$$

where $h_{\ell}$ is the Hopf invariant.

Taking the adjunction and quotients, we obtain a stable map

$$
f: S^{n+2 j+\ell} \rightarrow \Sigma^{\ell} D_{2}\left(\Sigma^{j} K\right) .
$$

As in the proof of 2.1, the composition given by $f$ followed by the transfer is a Spanier-Whitehead duality.

Using 3.3, we have $D_{2}^{\ell}\left(\Sigma^{j} K\right) \simeq \Sigma^{2 j} D_{2}^{\ell} K$ and this equivalence is compatible with transfers. Therefore, $f$ becomes identified with a stable map

$$
S^{n} \rightarrow D_{2}^{\ell} K
$$

which is an $\ell$-refined quadratic duality. 
Proof that (1) implies (2). Given $f: S^{d} \rightarrow D_{2}^{\ell} K$, let $j$ be large enough so that $\Sigma^{2 c-1} f$ exists as an ordinary map

$$
S^{d+2 c-1} \rightarrow \Sigma^{2 c-1} D_{2}^{\ell} K .
$$

We can assume that $c=e 2^{\phi(\ell-1)}$ for some $e \geq 1$. Compose now with $\omega_{\ell}$ to obtain a map

$$
\gamma: S^{d+2 c-1} \rightarrow \Sigma^{c} K
$$

and form its mapping cone $Y=\left(\Sigma^{c} K\right) \cup_{\gamma} D^{d+2 c}$. By 2.1, we infer that $Y$ is a Poincaré space whose top cell splits off after $\ell$-suspensions. Theorem A shows that $Y$ is $j$-periodic with $j=2^{\phi(\ell-1)}$.

\section{Appendix: the stable Hopf invariant Composition FORMULA}

In this section we will recall Crabb's definition [C, p. 62] of the stable Hopf invariant and use it to derive the formula for the Hopf invariant of a composition. We are including this material for the sake of completeness (as far as I know, the composition formula does not appear anywhere in the literature).

For based $\mathbb{Z}_{2}$-CW complexes $A$ and $B$, let

$$
[A, B]^{\mathbb{Z}_{2}}
$$

denote the associated set of equivariant homotopy classes of based $\mathbb{Z}_{2^{-}}$ maps. Let

$$
\{A, B\}^{\mathbb{Z}_{2}}
$$

be the colimit of $\left[S^{W} \wedge A, S^{W} \wedge B\right]^{\mathbb{Z}_{2}}$, where $W$ ranges over a complete universe of representations of $\mathbb{Z}_{2}$. Then $\{A, B\}^{\mathbb{Z}_{2}}$ is an abelian group.

If $X$ and $Y$ is a based CW complexes, there is a canonically split short exact sequence of abelian groups

$$
0 \longrightarrow\left\{X, D_{2} Y\right\} \stackrel{\delta}{\longrightarrow}\{X, Y \wedge Y\}^{\mathbb{Z}_{2}} \stackrel{\rho}{\longrightarrow}\{X, Y\} \longrightarrow 0
$$

where the map $\delta$ is induced by the transfer and $\rho$ assigns to an equivariant map $f: S^{W} \wedge X \rightarrow S^{W} \wedge Y \wedge Y$ the map

$$
f^{\mathbb{Z}_{p}}: S^{W^{\prime}} \wedge X \rightarrow S^{W^{\prime}} \wedge Y
$$

given by taking fixed points. Here $W^{\prime}$ is the fixed set of $W, X$ asnd $Y$ are given the trivial action and $Y \wedge Y$ has the permutation action. The splitting for $\rho$ is given by assigning to $X \rightarrow Y$ its composition with the reduced diagonal map $\Delta_{Y}: Y \rightarrow Y \wedge Y$.

The stable Hopf invariant of a stable map $f: X \rightarrow Y$ is the unique homotopy class $h(f): X \rightarrow D_{2} Y$ such that

$$
\delta(f):=(f \wedge f) \circ \Delta_{X}-\Delta_{Y} \circ f .
$$


Proposition 7.1. Let $f: X \rightarrow Y$ and $g: Y \rightarrow Z$ be stable maps. Then

$$
h(g \circ f)=D_{2}(g) \circ h(f)+h(g) \circ f .
$$

Proof. Consider the following maps

(1) $(g \wedge g) \circ(f \wedge f) \circ \Delta_{X}$,

(2) $(g \wedge g) \circ \Delta_{Y} \circ f$,

(3) $\Delta_{Z} \circ g \circ f$.

Then

$$
\begin{aligned}
\delta(h(g \circ f)) & =(1)-(3), \\
& =(1)-(2)+(2)-(3), \\
& =\delta\left(D_{2}(g) \circ h(f)\right)+\delta(h(g) \circ f), \\
& \left.=\delta\left(D_{2}(g) \circ h(f)\right)+h(g) \circ f\right) .
\end{aligned}
$$

Now use the fact that $\delta$ is injective to complete the proof.

\section{REFERENCES}

[A] Adams, J. F.: On the non-existence of elements of Hopf invariant one. Ann. of Math. 7, 220-104 (1960)

[BS] Boardman, J. M., Steer, B.: On Hopf invariants. Comment. Math. Helv. 42, 180-221 (1967)

[C] Crabb, M.C.: $\mathbb{Z} / 2$-homotopy theory. London Mathematical Society Lecture Note Series, 44. Cambridge University Press, 1980.

[H] Hodgson, J. P. E.: Question appearing the Problem Session. In: Proceedings of the Northwestern Homotopy Theory Conference (1982), Contemp. Math. 19, p. 448. AMS 1983

[HV] Hausmann, J. C., Vogel P.: Geometry on Poincar spaces. Mathematical Notes, 41. Princeton University Press, 1993.

[J] James, I. M.:Cross-sections of Stiefel manifolds. Proc. London Math. Soc. $8536-547$ (1958).

[K1] Klein, J. R.: Poincaré embeddings and fiberwise homotopy theory. Topology 38, 597-620 (1999)

[K2] Klein, J. R.: Embedding, compression and fiberwise homotopy theory. Alg. \& Geom. Topology 2, 311-336 (2002)

$[\mathrm{Ku}] \quad K u h n, \mathrm{~N} .:$ The geometry of the James-Hopf maps. Pacific J. Math. 102, 397-412 (1982)

[KR] Klein, J.R., Richter W.: Poincaré duality and periodicity. arXiv preprint (http://arxiv.org/pdf/0707.1353.pdf)

[Mh] Mahowald, M.: A short proof of the James periodicity of $\pi_{k+p}\left(V_{k+m, m}\right)$. Proc. Amer. Math. Soc. 16 p. 512 (1965)

[My] May, J. P.:The geometry of iterated loop spaces. (LNM Vol. 271). Springer, 1972

[Mi] Milgram, R. J.: Unstable homotopy from the stable point of view. (LNM, Vol. 368). Springer 1974

[R] Ranicki, A.: The algebraic theory of surgery. II Applications to topology. Proc. London Math. Soc. 40, 193-283 (1980) 
[S] Snaith, V.P.: A stable decomposition of $\Omega^{n} S^{n} X$. J. London Math. Soc. 7, 577-583 (1974).

[W1] Wall, C. T. C.: Poincaré complexes I. Ann. of Math. 86, 213-245 (1967)

[W2] Wall, C. T. C.: Surgery on Compact Manifolds. Academic Press 1970

Dept. of Mathematics, Wayne State University, Detroit, Mi 48202

E-mail address: klein@math.wayne.edu

Dept. of Mathematics, Northwestern University, Evanston, IL 60208

E-mail address: richter@math.northwestern.edu 